\title{
An Explanation on the Prevalence of Learning Disorders in Terms of Socioeconomic Factors of Health among the Elementary School Students in Larestan City during Year 2014
}

\author{
Eghbal Sekhavati ${ }^{1, *} ;$ Mojtaba Rahimian Boogar ${ }^{1}$; Sara Bostani ${ }^{1 ;}$;ahra Raeisi ${ }^{1}$ \\ ${ }^{1}$ Larestan school of Medical Sciences, Larestan, Iran \\ *Corresponding author: Eghbal Sekhavati, Larestan school of Medical Sciences, Larestan, Iran. Tel:+98- 7152342877 Email: sekhavati.papers@gmail.com
}

Received: 22 Sep 2016

Accepted: 21 Nov 2016

Ppub: 23 Dec 2016

\begin{abstract}
Background: Learning problems are expected to have different incidence rates depending on environmental factors. Nevertheless, the purpose of the present study is to explain the prevalence of learning disorders in terms of Socioeconomic Factors of health among the elementary school students in the city of Larestan during 2014.

Methods: In this descriptive- cross sectional study, 1800 students of grade two to six of the elementary school were selected as statistical samples of the research using the multi-step cluster sampling method and the questionnaires of socio-structural health determinants, Wechsler revised intelligence scale for children (WISC-R), the Colorado learning difficulties scale (CLDQ), as well as the clinical interview (Based on diagnostic and statistical guide of mental disorders) were completed. Finally, the data were analyzed using the regression logistic analysis, variance analysis, and the chi-square test.

Results: The general prevalence of learning disorders in the overall population of the subjects was $6.22 \%$ where learning disorders for dyslexia, writing disorder, and acalculia were $2.94 \%, 1.22 \%$, and $2.05 \%$, respectively. The findings revealed that the Socioeconomic Factors of health were significantly able to separate students with learning disorders from those without learning disorders $(\mathrm{p}<0.05)$ so that the strongest predictor of the occurrence chance of learning disorders in this model was the accessibility of health services of desirable quality with the odds ratio of 4.988 followed by poverty and disability in providing health costs with the odds ratio of 4.889 ( $\mathrm{p}<0.05)$. In other words, the students with learning disorders possess an inferior status of Socioeconomic Factors. Conclusions: The results of the present study showed that the Socioeconomic Factors of health make an important contribution to the occurrence and experience of mental disorders. Thus, these findings have practical and scientific consequences for the clinical experts in designing interventions and remedial training in learning disorders. Therefore, it is recommended that an operational plan is provided to reform, lift, or control the risk factors of the Socioeconomic Factors of health for those suffering from learning disorders regarding the target group at risk along with their prevention and treatment programs.

Keywords: Learning Disorders; Socioeconomic Factors; Health; Students
\end{abstract}

